PREPARED FOR THE U.S. DEPARTMENT OF ENERGY, UNDER CONTRACT DE-AC02-76CH03073

PPPL-3481

PPPL-3481

UC-70

IAEA Theory Summary 1998 IAEA Meeting,

Yokohama, Japan Oct. 17-24, 1998

by

W.M. Tang

August 2000

$=$ PPPL

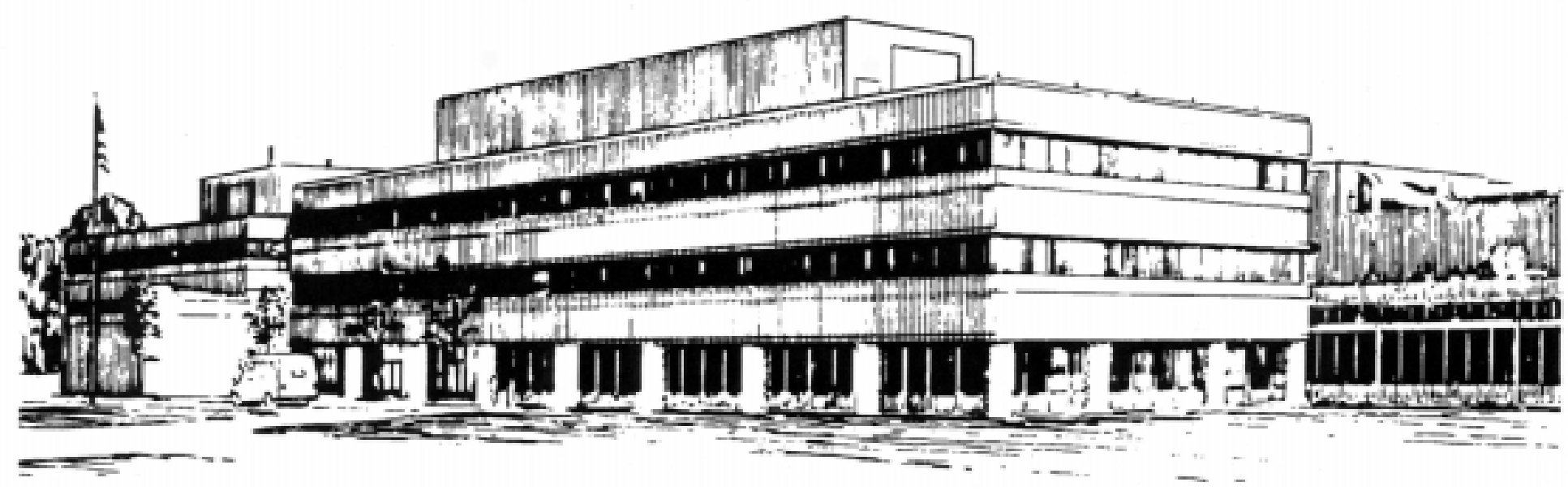

PRINCETON PLASMA PHYSICS LABORATORY PRINCETON UNIVERSITY, PRINCETON, NEW JERSEY 


\section{PPPL Reports Disclaimer}

This report was prepared as an account of work sponsored by an agency of the United States Government. Neither the United States Government nor any agency thereof, nor any of their employees, makes any warranty, express or implied, or assumes any legal liability or responsibility for the accuracy, completeness, or usefulness of any information, apparatus, product, or process disclosed, or represents that its use would not infringe privately owned rights. Reference herein to any specific commercial product, process, or service by trade name, trademark, manufacturer, or otherwise, does not necessarily constitute or imply its endorsement, recommendation, or favoring by the United States Government or any agency thereof. The views and opinions of authors expressed herein do not necessarily state or reflect those of the United States Government or any agency thereof.

\section{Availability}

This report is posted on the U.S. Department of Energy's Princeton Plasma Physics Laboratory Publications and Reports web site in Calendar Year 2000. The home page for PPPL Reports and Publications is: http://www.pppl.gov/pub_report/

DOE and DOE Contractors can obtain copies of this report from:

U.S. Department of Energy

Office of Scientific and Technical Information

DOE Technical Information Services (DTIS)

P.O. Box 62

Oak Ridge, TN 37831

Telephone: (865) 576-8401

Fax: (865) 576-5728

Email: reports@adonis.osti.gov

This report is available to the general public from:

National Technical Information Service

U.S. Department of Commerce

5285 Port Royal Road

Springfield, VA 22161

Telephone: $1-800-553-6847$ or

(703) $605-6000$

Fax: (703) 321-8547

Internet: http://www.ntis.gov/ordering.htm 


\title{
IAEA THEORY SUMMARY 1998 IAEA Meeting, Yokohama, Japan - Oct. 17-24, 1998
}

\author{
W. M. Tang \\ Princeton University, Plasma Physics Laboratory \\ Princeton, New Jersey, USA
}

\section{Introduction}

This is a summary of the advances in magnetic fusion energy theory research presented at the 17th International Atomic Energy Agency Fusion Energy Conference from 19-24 October, 1998 in Yokohama, Japan. Theory and simulation results from this conference provided encouraging evidence of significant progress in understanding the physics of thermonuclear plasmas. Indeed, the grand challenge for this field is to acquire the basic understanding that can readily enable the innovations which would make fusion energy practical. In this sense, as depicted in Fig. 1, research in fusion energy is increasingly able to be categorized as fitting well the "Pasteur's Quadrant" paradigm[1], where the research strongly couples basic science ("Bohr's Quadrant") to technological impact ("Edison's Quadrant"). As supported by some of the work presented at this conference, this trend will be further enhanced by advanced simulations. Eventually, realistic three-dimensional modeling capabilities, when properly combined with rapid and complete data interpretation of results from both experiments and simulations, can contribute to a greatly enhanced cycle of understanding and innovation. Plasma science theory and simulation have provided reliable foundations for this improved modeling capability, and the exciting advances in high-performance computational resources have further accelerated progress.

There were 68 papers presented at this conference in the area of magnetic fusion energy theory. They can be roughly categorized along their scientific areas of emphasis into five areas: (i) Turbulence and Transport [22 papers]; (ii) Macroscopic Equilibrium and Stability [20 papers]; (iii) Fast Particle Physics [9 papers]; (iv) Plasma Boundary Physics (10 papers); and (v) Wave/Plasma Interactions and Heating [7 papers]. As illustrated in Fig. 2, each of these areas individually provide major research challenges, but the ultimate goal will be to produce an effectively integrated physics-based modeling capability (encompassing key physics from all of these areas collectively) that will be able to predict fusion device performance with great reliability. This will require strong coupling to experiments and, if successful, would lead to prominent benefits such as (i) more cost-effective utilization of present-generation facilities to explore tokamak and alternate concepts; and (ii) accelerated progress to better designs for future devices. 


\section{Considerations of Use?}

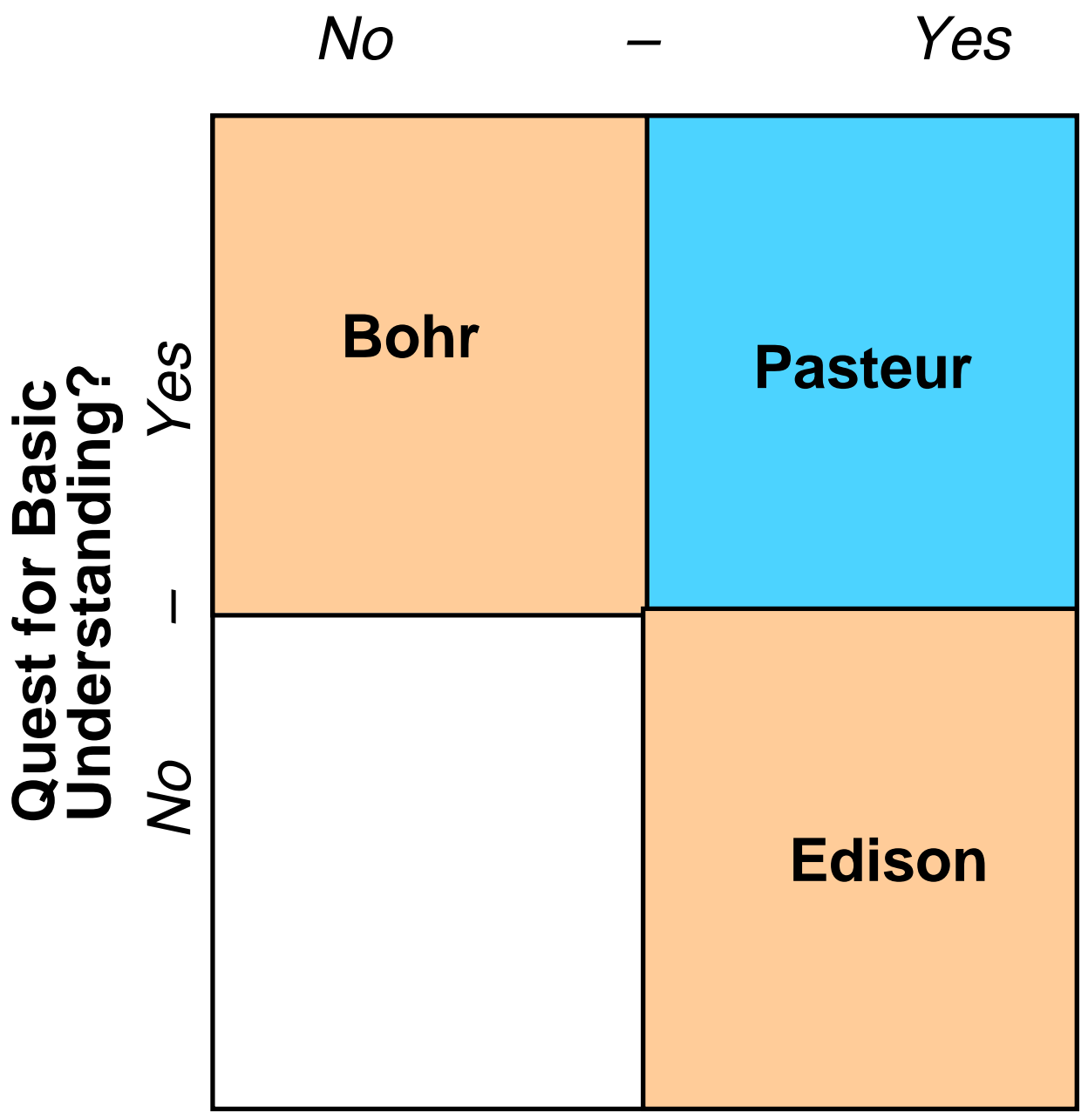

Figure 1: Fusion Energy Science in Pasteur's Quadrant

\section{Research Advances}

This section highlights the challenges, progress, and future objectives in each of the key topical areas depicted in Fig. 2. Each of these is a fascinating area of science in its own right, as well as critical for the development of fusion power. The basic goal in the associated research is the advancement of fundamental understanding of magnetically-confined plasmas to enable enhancement of predictive capabilities for fusion-grade plasmas. This requires effective interactions/comparisons of experiment, theory and simulation.

\subsection{Turbulence and Transport}

The goal of understanding turbulence and the associated transport is not only a fundamental issue of general scientific importance but is also critical for attaining adequate gain in fusiongrade plasmas. As reported at this conference, excellent progress has been made in associating the the ion-temperature gradient (ITG) mode with anomalous heat transport in the ion channel. The advances in computational capabilities have enabled major progress in predicting the nonlinear consequences of these key instabilities. Knowledge about the electron channel would need to reach a similar level of maturity, before a robust predictive capability based 


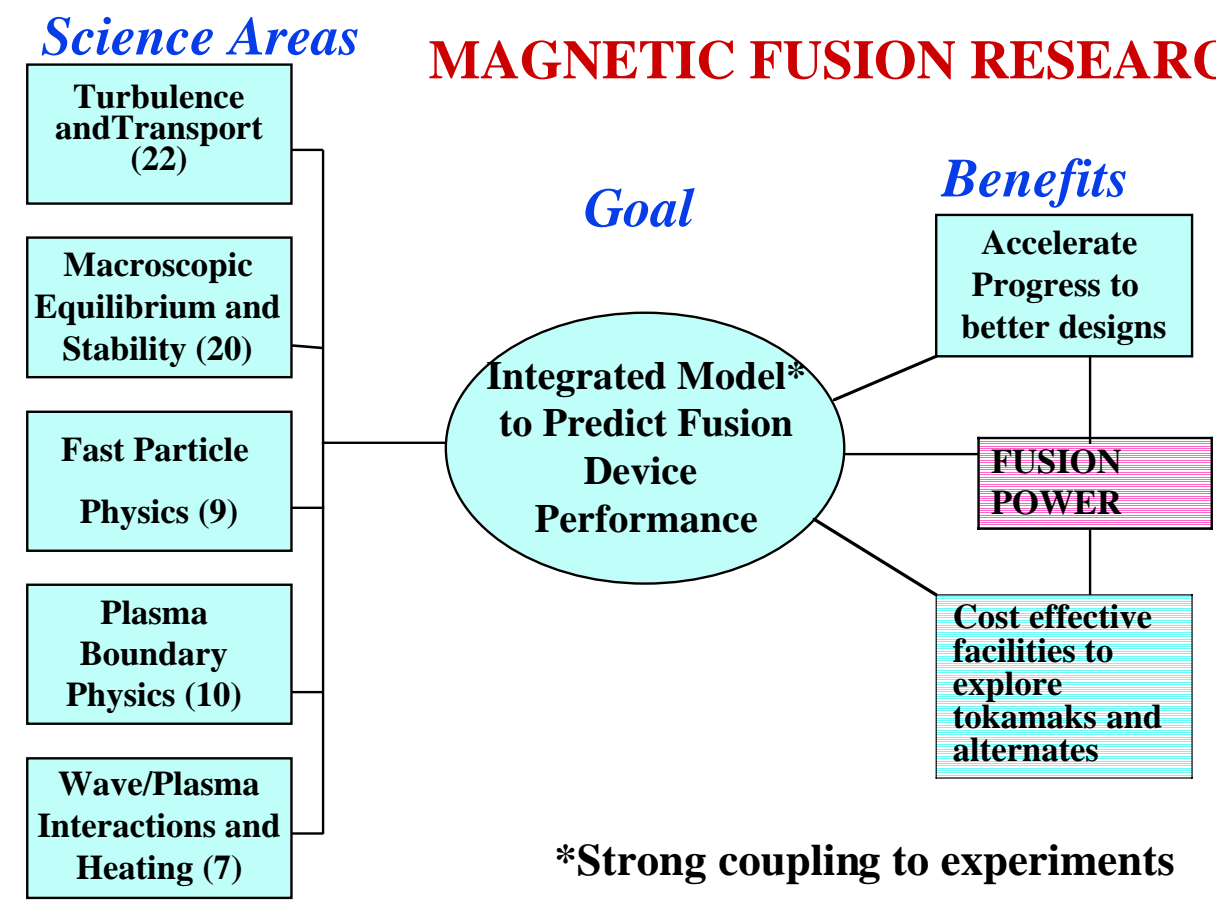

Figure 2: Scientific Areas, Goal, and Benefits in MFE Theory Research

on first-principles physics could be expected. Achieving this goal will also require the active collaborative participation of well-diagnosed major experimental facilities to challenge and/or corroborate the theoretical models.

The major challenges in this area include: (i) realism with respect to physics and geometry; (ii) interpretive and predictive capability [e.g., scaling trends, confinement transition thresholds, ...]; and (iii) effective applications to key experimental questions and design issues. Progress in linear theory, which provides the tools to assess the onset conditions for instabilities that inhibit plasma performance, featured the inclusion of $\mathbf{E} \times \mathbf{B}$ flow and realistic geometric effects. Successful applications to high-confinement experimental scenarios were reported [e.g., papers THP2/13, 19, 28] along with optimistic projections for future low-aspect-ratio, high-beta devices [TH1/4]. However, such predictions of "neoclassical transport levels" need to be tempered by the fact that self-consistent assessments of macroscopic stability for these cases have yet to be carried out.

Progress in nonlinear theory, which deals with the much more challenging task of assessing the consequences of instabilities on confinement, included: (i) possible "supercritical" (above-linear-threshold) behavior in radially-local ("flux-tube") gyrokinetic simulations of turbulence due to ion-temperature gradient driven microinstabilities [paper TH1/1] and "subcritical" (below-linear-threshold) behavior in fluid simulations of flux-driven turbulence [paper TH1/3]. In the latter paper, the fluid simulations for a fixed flux (instead of the more conventional fixed gradient) model exhibited avalanches and dynamics characteristic of self-organized critical behavior with respect to intermittency and spectral features. In the sense that the work in this area exhibited the best examples of synergism between analytic theory, advanced simulations, and experiments, the most prominent area of progress dealt with the physics of flow shear suppression of turbulence [e.g, papers TH3/1, THP2/28, TH1/1, THP2/12]. Attention was focused on zonal flows - which are basically sheared $\mathbf{E} \times \mathbf{B}_{0}$ flow layers generated by the modulation of radial current induced by microturbulent (e.g., drift-wave type) fluctuations. In the descriptive "predator-prey" picture, the zonal flows ("predator") act to suppress and/or 
saturate the microturbulence ("prey"). This is a self-regulating process in which the flows are asymptotically damped by collisions. The dynamical properties are being explored with advanced gyrofluid [TH1/1] and gyrokinetic [THP2/28] simulations. In the latter paper, a new general-geometry 3-dimensional gyrokinetic code, GTC, has enabled realistic neoclassical and turbulent transport studies to be successfully carried out on powerful new massively-parallel processor (MPP) computers that have recently become accessible. These advanced simulations, which could not have been carried out even one year ago, systematically demonstrated for the first time that substantial reduction of transport is due to turbulence-generated $\mathbf{E} \times \mathbf{B}_{0}$ zonal flows - thereby corroborating key trends from analytic estimates and experimentally-observed trends. As depicted in Fig. 3, this work provides an effective illustration of how advanced scientific computing can be an valuable new "tool for discovery" complementing theory and experiment.

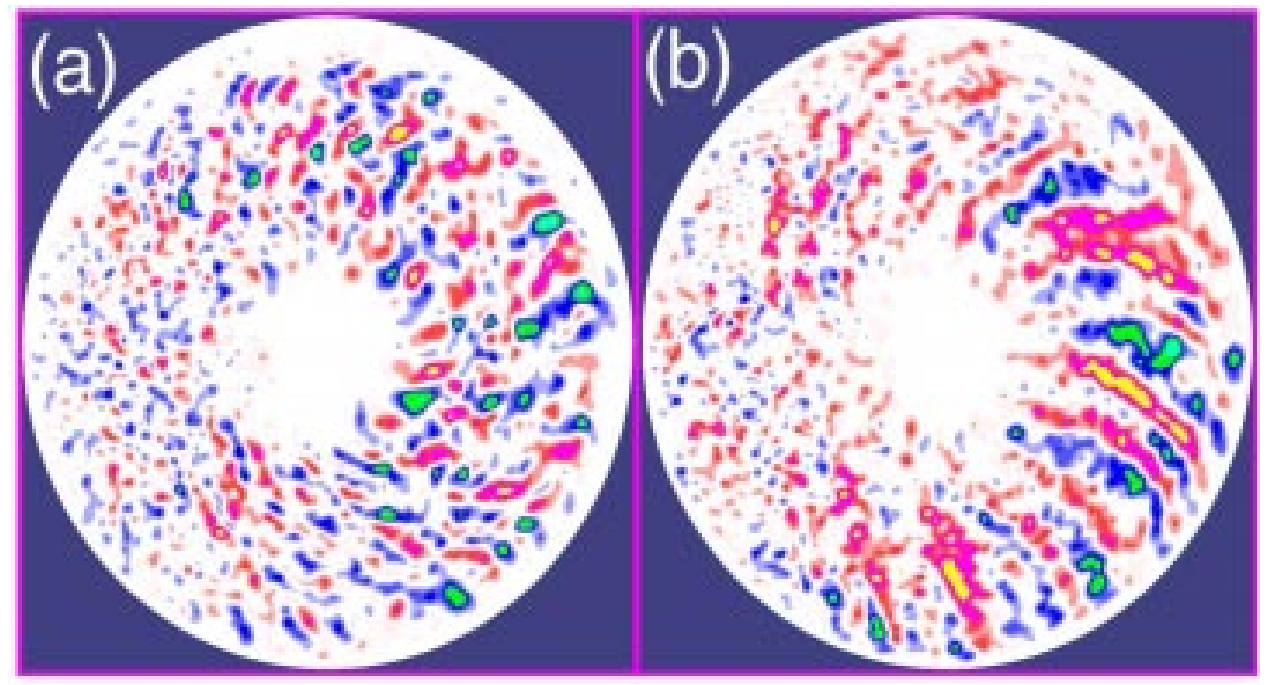

\section{With Flow}

\section{Without Flow}

Figure 3: Three-dimensional Particle Simulations of Zonal Flow Effects

With regard to the modeling of transport barriers and the modeling of general transport trends in toroidal experiments, the progress reported included observations on the possible relevance of discontinuous drift-wave mode characteristics for internal transport barriers [paper TH1/2]. A model based on current-diffusive ballooning modes was found to account for features of transport barrier formation and collapse [paper TH1/6]. Although the applications aspect of these results were quite compelling, a strong justification of the robustness of the current-diffusive ballooning modes via nonlinear analysis and simulations remains elusive. Applications of a multi-mode predictive transport code with additional physics features recently incorporated have produced improved correlations with a number of key tokamak experiments [paper THP2/19]. Finally, results from semi-empirical models were found to correlate with confinement trends observed in JT-60U [THP2/17] and with confinement scaling in the stellarator experiment, W7-AS [THP1/5], as well as in JET [THP2/18].

Future goals in the key area of turbulence and transport research include: (i) understanding the relationship between advanced gyrokinetic global and radially local ("flux-tube" type) simulations and also for global (e.g., paper THP2/06) versus radially local gyrofluid models; (ii) 
increased applications to alternate confinement systems including Stellerators, Spherical Tori (ST's), Reversed field Pinches (RFP's), Field-Reversed Configurations (FRC's), ..., as well as to advanced tokamaks; and (iii) improve interpretive and predictive capability by including much-needed key physics [such as complete electron dynamics, full electromagnetic ("finite beta") physics, general 3-dimensional geometry, ... ] properly benchmarked against analytic estimates and experiments. Generally, research in the years ahead will strive for progress in understanding of turbulent transport to the level where theoretical predictions can be confidently viewed as being more reliable than empirical scaling in the leading experimental devices.

\subsection{Macroscopic Equilibrium and Stability}

The current status of research in magnetohydrodynamics (MHD), which encompasses fusion device scale (macroscopic) physics, is that predictions based on ideal MHD, in which smallscale dissipative effects are ignored, give excellent predictions of very fast time scale phenomena. However, a physics-based predictive capability for the slower time scale macroscopic phenomena, which result from dissipative (resistive and kinetic) effects, remains a formidable challenge. Indeed, controlling the macroscopic stability of plasmas in the presence of these small-scale resistive and kinetic dynamics is key to achieving and sustaining high pressure plasmas. For example, under certain conditions the neoclassical tearing mode can produce a slow breakup of magnetic surfaces at moderate plasma pressures well below the ideal thresholds in tokamaks. The theoretical foundations for an effective quantitative analysis of this important instability needs to be developed. Possible alleviation of this problem with appropriately localized pressure gradients driven by electron-cyclotron resonant heating is being actively pursued [TH2/5]. More generally, physics insights from experiments are vital to timely progress on key issues such as feedback stabilization of dissipative modes in tokamaks, high beta limits in spherical tori, and both stabilizing and destabilizing effects of resistive dynamics in devices including Stellarators, Reversed Field Pinches, and Field Reversed Configurations.

It is generally acknowledged that a major obstacle to the reliable sustainment of magnetic confinement is the presence of disruptions - the occurrence of sudden and dramatic loss of plasma confinement at critical values of the plasma pressure and/or the plasma current. Progress reported at this meeting included results from nonlinear three-dimensional MHD simulations of the current quench phase of disruptions in tokamaks with associated halo current and runaway electron behavior [TH3/4]. With regard to other configurations, internal disruptions observed in Heliotrons were related to the nonlinear evolution of ideal MHD modes $(\mathrm{m} / n=2 / 1)$ [THP1/4], and the kinetic stabilization of tilt disruptions in Field Reversed Configurations from three-dimensional particle simulations were reported in paper [THP1/11]. Macroscopic equilibrium and stability analysis capabilities have also been effectively applied to performance improvement studies in presently operating experiments and for the design of new configurations - advanced tokamaks as well as alternative magnetic confinement concept systems. A representative example is the investigation of compact quasi-axisymmetric stellarators. In paper [THP1/7] it is reported that equilibrium and stability analyses indicate that such configurations can have a high bootstrap current fraction and also a reasonably high ideal MHD beta limit without a conducting wall.

The interpretation of important macroscopic phenomena observed in tokamak and alternative magnetic confinement experiments was highlighted in several papers. For example, the importance of accounting for flow shear effects in assessing experimental trends observed in tokamaks [THP2/30], [TH3/7] and also for Reversed Field Pinches [THP1/10] was emphasized in several papers. Insights into the relative role of resisitive interchange instabilities were 
obtained from comparative linear studies of such modes for tokamaks in regions of negative magnetic shear and for heliotrons [TH2/2]. In the area of Spherical Torus (ST) studies, paper [TH3/2] concluded that in the Compass-D experiment, the MHD peeling mode could provide an explanation for the observed low density L-H transition threshold and possibly also for the edge localized mode (ELM) behavior. Significant progress in understanding internal reconnection dynamics in ST's was provided by impressive three-dimensional nonlinear MHD simulations [TH3/3] which addressed several key features observed in such systems. The basic picture proposed is that the nonlinear development of ballooning modes produces pressure bulges that can drive magnetic reconnection. Reconnection occurs between field lines located inside the separatrix and those outside, thereby allowing heat to escape along the reconnected field lines. As depicted in Fig. 4, this work provides another effective illustration of how advanced scientific computing can be a valuable new "tool for discovery" complementing theory and experiment.

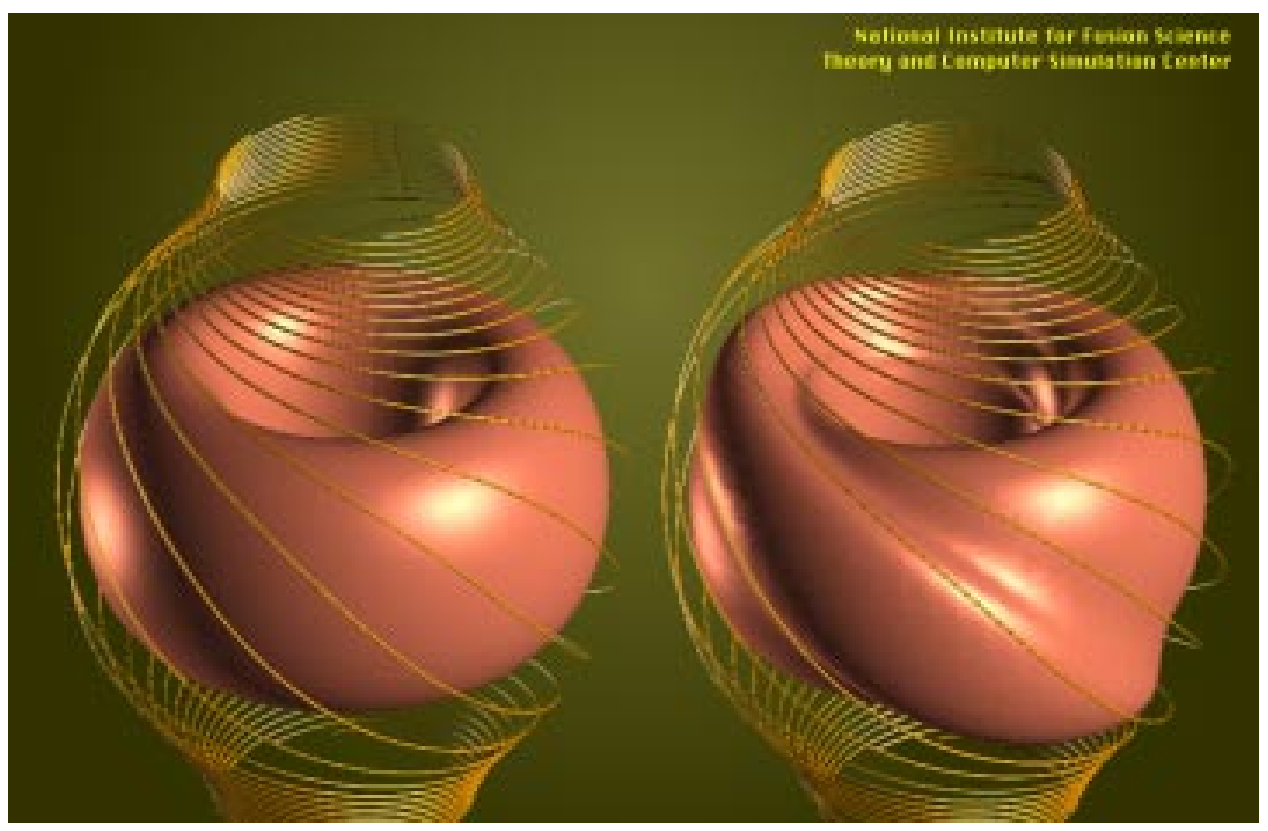

Figure 4: MHD Simulation of Internal Reconnection Event in a Spherical Torus

\subsection{Fast Particle Physics}

Understanding the behavior of fast/energetic particles in magnetically confined devices is crucial to the effective assessment of their relevance to alpha-particle physics issues in present and future D-T plasmas. This area of research has witnessed excellent achievements in first predicting and then experimentally identifying key instabilities such as the toroidal Alfven eigenmodes in prominent experiments such as TFTR, JET, JT-60U, .... The primary challenge is the development of a realistic predictive capability for energetic- particle-driven instabilities in reactor-relevant regimes. This will require: (i) more realistic physics descriptions including general geometry and non-perturbative treatment of kinetic dynamics for both thermal and fast particles; and (ii) active applications to tokamaks as well as alternative magnetic confinement experiments including stellarators and spherical tori. If effectively exploited, important experimental research opportunities that would help stimulate and drive theoretical efforts in this area include possible future DT campaigns on JET and the high-energy negative-ion neutral beam 
program on JT-60U. This would of course also be a key topic of scientific investigation in future burning plasma experiments.

Progress in theoretical studies of fast particle physics reported at this conference included: (1) prediction of new Alfvenic ion temperature gradient (ITG) eigenmodes which were found to be unstable well below ideal ballooning threshold [TH2/3]; and (2) the development of a new non-perturbative code to analyze toroidal Alfven eigenmodes (TAE's) in the moderate to shortwavelength regime relevant to energetic particle effects in large plasmas [THP2/21]. In more direct applications to present experiments, Monte Carlo simulations indicating radial convective transport of suprathermal electrons were used to interpret the broadening of electron cyclotron resonant heating (ECRH) deposition profiles observed in the W7-AS stellarator [TH2/1]. In the JET experiment, compelling explanations for the observed bursting phenomena and frequency chirping have been given in terms of strongly nonlinear resonant wave-particle interactions. Specifically, a nonlinear theory, which includes a generalization of Landau damping, has been developed and applied to the interpretation of the "pitchfork-splitting" of TAE's in JET [TH2/4].

\subsection{Plasma Boundary Physics}

Understanding plasma boundary physics, which includes plasma-wall interactions and turbulent transport in the plasma edge region, is a formidable challenge facing magnetic fusion. Advances in the capability to reliably predict interactions between the plasma and its material interfaces have come primarily in tokamak divertor research. Improvements here will require: (1) formulation of the basic equations describing complex experimentally-observed processes such as filamentation and bursting behavior; and (2) generalization of present laminar models to more realistic three-dimensional descriptions. The latter is again a major task that will require strong interplay between advanced computing and benchmarking against theoretical models motivated by experimental trends. With regard to transport at the plasma boundary, the challenge is to develop a better physics description of edge turbulence. This is of course linked to most of the issues encountered in the core turbulence and transport area discussed earlier and is illustrative of the integration goal depicted in Fig. 2.

Progress in the divertor modeling area highlighted at this conference included: (1) results from applications to the ASDEX Upgrade tokamak of the B2-Eirene (coupled edge physics neutrals codes) indicated an improved predictive capability [THP2/05]; (2) possible influence of an ergodic divertor on plasma behavior at the plasma boundary [TH3/5]; (3) insights into detached plasma behavior in the divertor scrape-off layer of tokamaks [THP2/11]; and (4) a good example of improved physics insights on the key issue of tokamak divertor detachment and stability gained from productive interactions between theory, modeling, and experimental analysis [TH3/6]. As illustrated on Fig. 5, it is first noted that experimental results [EX3/3] indicate that, without feedback, the plasma recombination region moves toward the X-point as detachment proceeds. This motion can be interpreted in terms of the limits on the upstream plasma density, $n_{u p}$, which are set by both plasma recombination and impurity radiation effects. Upon reaching such limits, $n_{u p}$ saturates as the total number of particles in the scrape-offlayer, $N_{S O L}$, and the dense cold plasma and neutral gas region builds up in the divertor. As shown in the bottom panel of this figure, results from the UEDGE modeling code indicate that consistent with the experimental trends, this density build-up pushes the neutral ionization, impurity radiation, and recombination fronts to the $\mathrm{X}$-point.

Advances toward an improved description of edge turbulence were reported in several papers with the primary emphasis on fluid turbulence simulations at the tokamak boundary. Notable was the fact that reasonable agreement with key trends observed in the ASDEX-U tokamak 

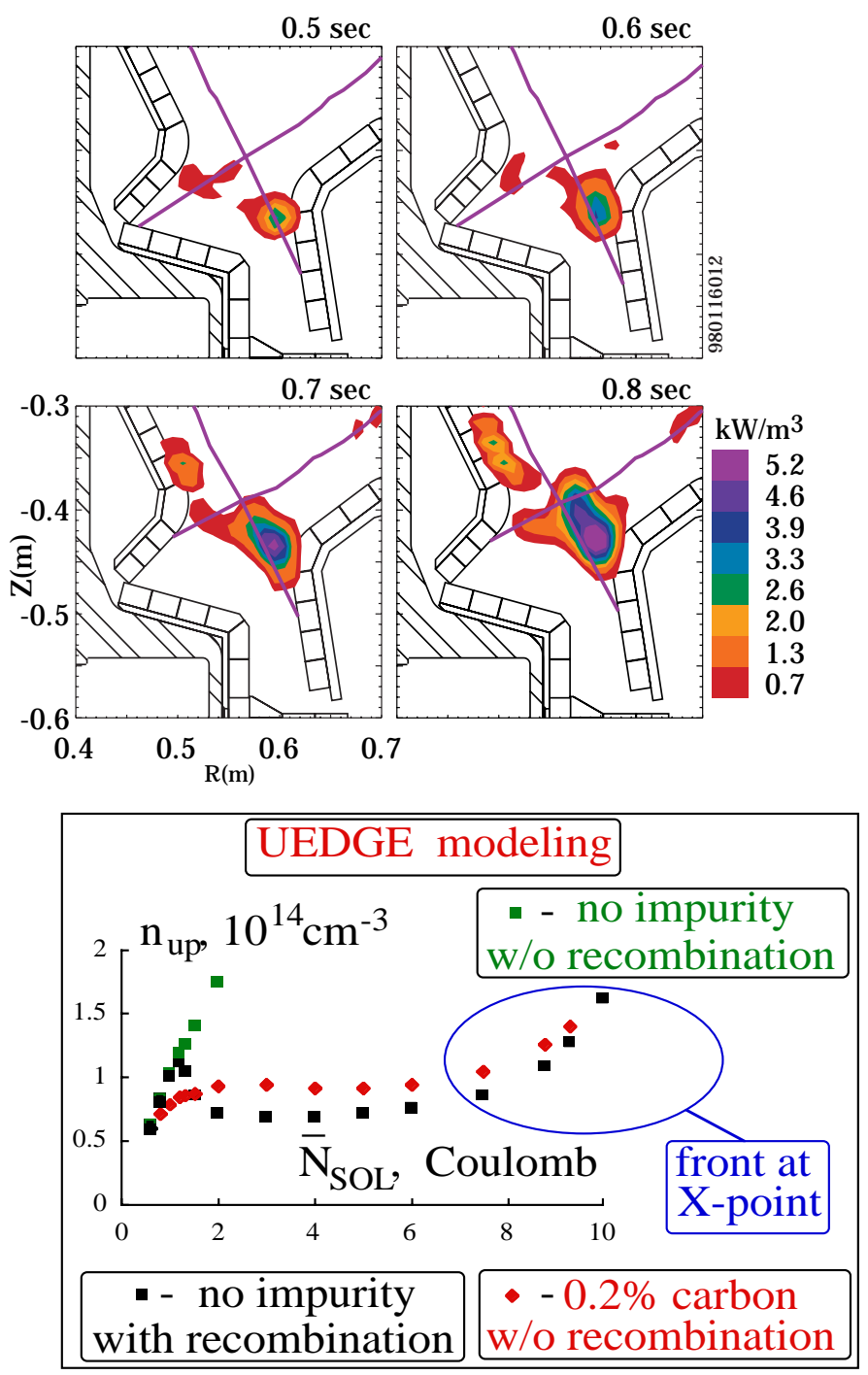

Figure 5: Tokamak Divertor Detachment Simulation

was reported in two separate studies using two significantly different models [TH1/5, TH1/7]. In order to consolidate progress in this area, reconciliation in the physics content and/or the computational algorithms utilized in the respective models is clearly needed. Results from studies of edge transport taking into account (i) realistic divertor geometry [THP2/03] and (ii) possible coupling to core physics trends [THP2/04] indicated that radially non-local analysis is needed to properly model $\mathrm{L} / \mathrm{H}$ transition dynamics.

\subsection{Wave/Plasma Interactions and Heating}

Development of a realistic predictive capability for plasma heating, flow, and current drive remains a difficult challenge in magnetic fusion research. Papers at this conference focused primarily on progress toward a better understanding of the relationship between wave heating of plasmas and rotational dynamics [TH3/7, THP2/34 through 37]. This requires first developing a self-consistent formulation of the radial electric field equation which includes its effect on turbulent momentum transport. As evident in the results highlighted in the turbulent transport part of the present summary, this is a key issue because of the role of flow shear for enhanced 
confinement and also because of its potential impact on profile control [THP1/12]. With regard to the prospect of significant enhancement of plasma performance enabled by various current drive profile control methods (e.g., ECCD and LHCD), improved RF models are clearly needed to better assess the efficacy of such approaches.

\section{Concluding Comments}

Theory and simulation results presented at this conference give evidence of significant advances in understanding the physics of thermonuclear plasmas. Much of the work has concentrated on interpreting existing experiments, and the achievements on this front are indeed encouraging. In the "Pasteur's Quadrant" sense noted earlier, further progression of these capabilities is expected to provide the fundamental knowledge needed for reliable quantitative predictions that can guide the way to improved plasma performance and to the innovative design of improved magnetic confinement devices. Accelerated progress toward this goal is now accessible via the exciting advances in information technology. If properly cultivated, this path can also help attract, train, and assimilate young talent essential for the future health of this field. As already evident in a number of the papers presented at this conference, advanced computing, when strongly linked to experiment and theory, can be a powerful new tool for discovery.

\section{Reference}

[1] Donald E. Stokes, "Pasteur's Quadrant: Basic Science and Technological Innovation," Brookings Institution Press, Washington, D. C. (1997) 
The Princeton Plasma Physics Laboratory is operated by Princeton University under contract with the U.S. Department of Energy.

\author{
Information Services \\ Princeton Plasma Physics Laboratory \\ P.O. Box 451 \\ Princeton, NJ 08543
}

Phone: 609-243-2750

Fax: 609-243-2751

e-mail: pppl_info@pppl.gov

Internet Address: http://www.pppl.gov 